American Journal of Applied Sciences 5 (10): 1285-1290, 2008

ISSN 1546-9239

(C) 2008 Science Publications

\title{
Dynamic Stress Analysis of the Arterial Wall Utilizing Physiological Pressure Waveforms
}

\author{
${ }^{1}$ M.M. Khani, ${ }^{1}$ M. Tafazzoli-Shadpour, ${ }^{1}$ S. Delavarpour, ${ }^{2}$ Sh. Naghizadeh and ${ }^{3}$ A. Avolio \\ ${ }^{1}$ Biomedical Engineering Faculty, \\ Amirkabir University of Technology (Tehran Polytechnic), Tehran, Iran \\ ${ }^{2}$ Material Engineering Faculty, Tarbiat Modares of University, Tehran, Iran \\ ${ }^{3}$ Australian Schools of Advanced Medicines, Macquarie University, Sydney, Australia
}

\begin{abstract}
Arterial diseases, promoted by alterations in arterial wall properties, are among the main causes of mortality. Mechanical stresses of the arterial wall caused by pulsatile luminal pressure define arterial function in normal and pathological conditions. This study aims to determine dynamic stress distribution in the arterial wall subjected to physiological pressure waveforms. Finite element models of a typical artery are developed to evaluate Von Misses stress in the arterial wall due to physiological pressure waveforms and with differing mechanical properties. Mechanical parameters include Young's modulus of elasticity, non-linear stress-strain relationship and visco-elastic parameter. The appropriate boundary conditions are allocated to allow radial expansion. Application of physiological pulsatile pressure results in stress waves with the values and waveforms markedly influenced by mechanical properties of the arterial wall and blood pressure pulse. Elevated elastic modulus of the arterial wall results in significant increase in maximum stress. Viscoelastic property leads to reduction of the peak stress and smoothening of the stress waveform. The pressure waveform is also a major parameter affecting the stress pattern in arterial wall. Hypertensive arteries result in higher and sharper stress waves not only because of a higher systolic value but also because of the sharper waveform. The combination of these parameters produces the resultant stress pattern in the arterial wall.
\end{abstract}

Key words: Dynamic stress, finite element, physiological pressure, arterial wall

\section{INTRODUCTION}

Cardiovascular mechanics is a major descriptor of pathology of cardiovascular diseases, especially atherosclerosis, which is a major contributor to cardiovascular morbidity and mortality ${ }^{[1,2]}$. One of the major fields of research in cardiovascular biomechanics is study of the effects of mechanical parameters on endothelial damage leading to atherosclerosis. The major parameters include hemodynamic pulsatile shear stress $^{[3-6]}$ and arterial wall pulsatile tensile stress ${ }^{[7-10]}$.

Results of previous studies have shown that excessive arterial wall tensile stress over a time span may lead to endothelial damage and consequently atherosclerotic plaque formation ${ }^{[11-15]}$. Static stress analysis has been considered to locate sites of tensile stress concentration ${ }^{[11,12]}$. These studies have not considered the fatigue process during time span and consequent altered stress pattern with time. Such effect necessitates dynamic stress analyses of the arterial wall.
This study aims to conduct dynamic stress analysis of the arterial wall subjected to physiological arterial pressure waveforms. In addition to parameters related to static stress, additional parameters are presented in dynamic stress analysis such as stress wave amplitude and shape and maximum/minimum values of stress pulse. Sensitivity analyses are performed to study effects of loading conditions and mechanical parameters on stress parameters. The loading parameters include the pressure waveform and values and major mechanical parameters include wall elasticity and viscoelasticity. Such parameters are altered in situations such as hypertension and aging. Results of this study may contribute to the understanding of underlying mechanical causes of atherogenesis and arterial wall degeneration.

\section{MATERIALS AND METHODS}

Finite element dynamic stress analysis is performed using ANSYS version 10 considering

Corresponding Author: Dr. M. Tafazzoli Shadpour, Biomedical Engineering Faculty, Amirkabir University of Technology (Tehran Polytechnic), Tehran, Iran Tel: =98-21-64542385 Fax: +98-21-66468186 
Am. J. Applied Sci., 5 (10): 1285-1290, 2008

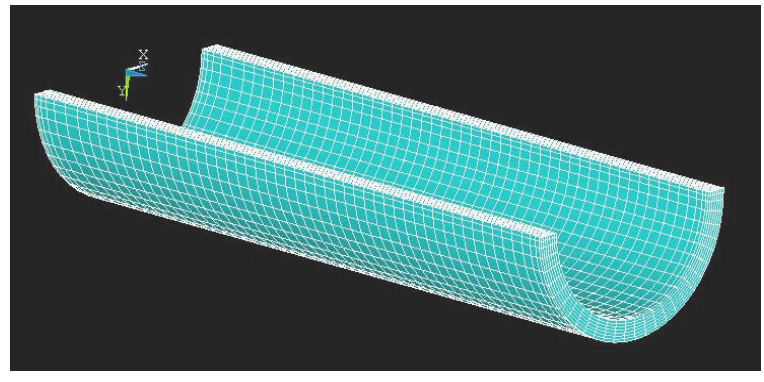

Fig. 1: Finite element model of arterial wall

viscoelastic properties of the arterial wall. A typical artery is modeled with internal and external diameters of 10 and $11 \mathrm{~mm}$, respectively (Fig. 1). The constraints of the model allow expansion in the radial direction.

Mechanical properties are allocated for component materials including Young's modulus of elasticity (E), viscoelastic parameter (C/E), Poisson's ratio and density. Sensitivity analyses are performed to investigate effects of change in mechanical properties. The viscoelastic parameter is defined as the ratio of the lost modulus (C) described by the viscous parameter to storage modulus (E) described by Young's modulus of elasticity. This parameter is elevated from 0 to $15 \%$ to investigate effects of viscoelasticity on the pulsatile stress value and waveform. The range of alteration of the viscoelastic parameter is in accordance with those of soft biological tissues ${ }^{[16,17]}$.

To analyze effects of wall stiffness, the elastic modulus is elevated from 300 to $900 \mathrm{kPa}$ in different steps. Such range describes elastic modulus of major elastic arteries ${ }^{[18]}$. Due to small values of elastic moduli, large deformation algorithm is set for proper expansion of the arterial wall in radial direction.

A pulsatile luminal pressure is applied on the intimal side of the model with the waveform and duration of typical arterial pulses. Three different pressure waves are presented based on physiological data (Fig. 2) with specific waveforms, duration and systole/diastole values ${ }^{[19,20]}$. Such pressure pulses include a normal subclavian artery pressure wave (Fig. 2a) and normal and hypertensive ascending aortic pressure waves (Fig. 2b, c).

To apply the dynamic pressure pulse on the model, a static load equivalent to mean value is applied first and then the pressure wave is simulated. The pulsatile loading is applied for five cycles to allow a proper convergence of results and to overcome the transient effects. The resultant stress waves are obtained from the fifth pulse. The criterion of convergence is applied for the results in such a way that the difference between maximum values of the last two stress waves is smaller

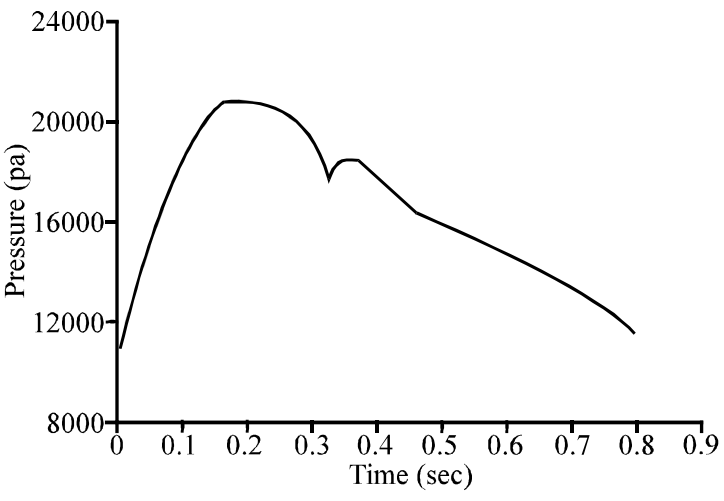

(a)

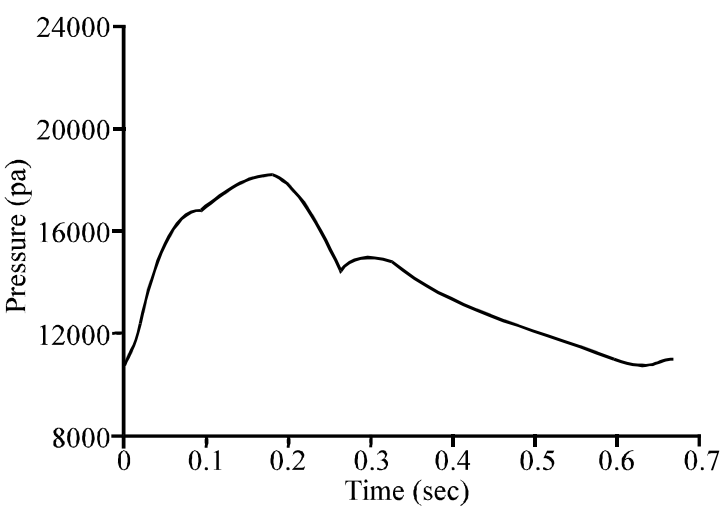

(b)

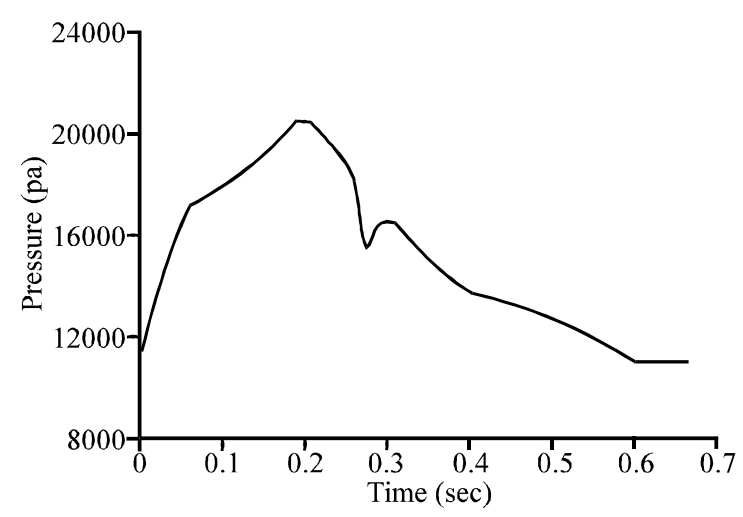

(c)

Fig. 2: Applied pressure pulses: (a): normal subclavian artery pressure waves, (b): normal ascending aortic pressure wave and (c): hypertensive ascending aortic pressure wave

than the set tolerance. After allocation of mechanical properties and pressure waveforms to the models, Von Misses stress distributions across the arterial thickness are obtained and compared for differing mechanical properties and pressure waveforms. 
The resultant stress parameters include stress waveform, the maximum/ minimum stress values of the stress wave and stress profiles across the arterial thickness.

\section{RESULTS AND DISCUSSION}

Effects of elastic modulus: To investigate effects of wall elasticity, resultant stress values are presented for models with differing elastic moduli without consideration of viscoelastic property. Results indicate that for all models, the maximum Von Misses stress occurs at the intima side. The resultant stress is pulsatile. The stress waveform and minimum/maximum values $\left(S_{\min }\right.$ and $\left.S_{\max }\right)$ depend on wall mechanical properties. Sensitivity analyses reveal that the stress distribution is not significantly sensitive to the Poisson's ratio and density values.

Figure 3 indicates effects of change in elastic modulus on the stress pattern. Figure $3 \mathrm{a}$ shows the profile of peak of stress wave across the wall thickness for an applied normal aortic pressure waveform with different elastic module and $\mathrm{C} / \mathrm{E}=0$. The stress profile is linear with maximum and minimum values on intima and adventitia sides respectively. The linear behaviour can be attributed to the lack of viscoelasticity and the small thickness/diameter ratio. With increase of $\mathrm{E}$ from 300 to $900 \mathrm{kPa}$ an increase of $18 \%$ in maximum stress pulse is observed. The reduction in maximum stress value from intima to adventitia is $20 \%$ for $\mathrm{E}=300 \mathrm{kPa}$ and $17.5 \%$ for $\mathrm{E}=900 \mathrm{kPa}$. Figure $3 \mathrm{~b}$ indicates the non-linear increase in maximum per minimum stress values with increasing $E$ in a model with subclavian pressure waveform. By elevation of elastic modulus from 300 to $900 \mathrm{kPa}$ the maximum and minimum stress values are elevated 15.6 and $23.4 \%$, respectively.

Effects of viscoelasticity: To investigate effects of viscoelastic behaviour on the stress pattern, the viscoelastic parameter $(\mathrm{C} / \mathrm{E})$ with four different values is included as the material property of the model. Figure $4 \mathrm{a}$ shows, the stress profile indicating the peak stress value on stress wave along the arterial wall thickness for an applied aortic pressure waveform with $\mathrm{E}=300 \mathrm{kPa}$ and differing viscoelastic parameter. Compared to the results shown in Fig. 3a, there is a reduction of stress level from intima to adventitia with a non-linear trend. Due to viscoelastic related energy dissipation of a viscoelastic material, the strain energy and consequently, the stress values are reduced compared to the material with the same elastic modulus and no viscoelastic behaviour (Fig. 3a).

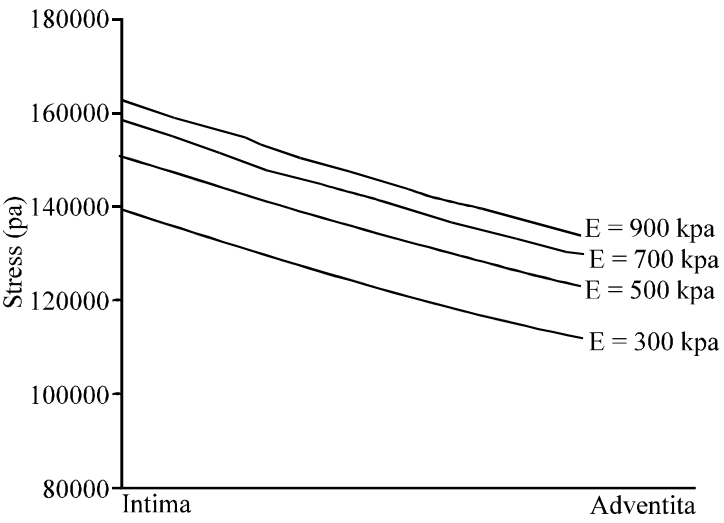

(a)

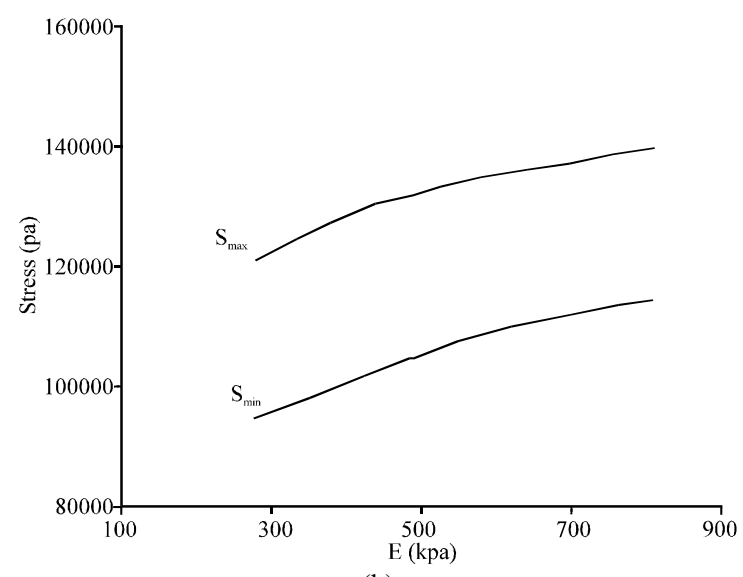

(b)

Fig. 3: Effects of wall elastic modulus on dynamic stress: (a): profile of maximum stress across the arterial wall (b): maximum and minimum stress values on intima

Figure $4 \mathrm{~b}$ shows the maximum and minimum values of the stress wave in a model with $E=300 \mathrm{kPa}$ and differing viscoelastic modulus. The maximum and minimum values are related to systolic and diastolic pressures. The amplitude of the resultant stress wave decreases with increasing viscoelastic modulus. Results show that by increase of $\mathrm{C} / \mathrm{E}$ from 2 to $15 \%$, almost $10 \%$ of the maximum stress value and $41 \%$ of the amplitude of stress pulse decrease. On the other hand, the ratio of minimum stress to maximum stress $\left(\mathrm{R}=\mathrm{S}_{\min } / \mathrm{S}_{\max }\right)$ increases from $65 \%$ for $\mathrm{C} / \mathrm{E}=2$ to $47 \%$ for $\mathrm{C} / \mathrm{E}=2 \%$.

Effects of viscoelasticity are stressed in Fig. 5, in which the two stress waves from an identical pressure wave are presented. Alteration in stress waveform is solely due to viscoelatic property. Viscoelastic behaviour not only results in a reduction of maximum stress value but also causes smoothening of the 
Am. J. Applied Sci., 5 (10): 1285-1290, 2008

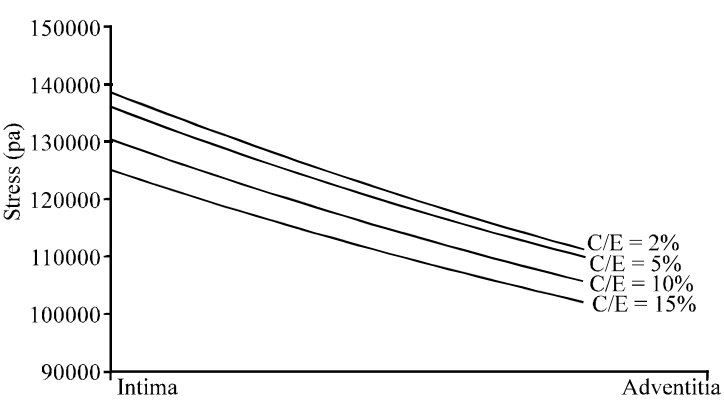

(a)

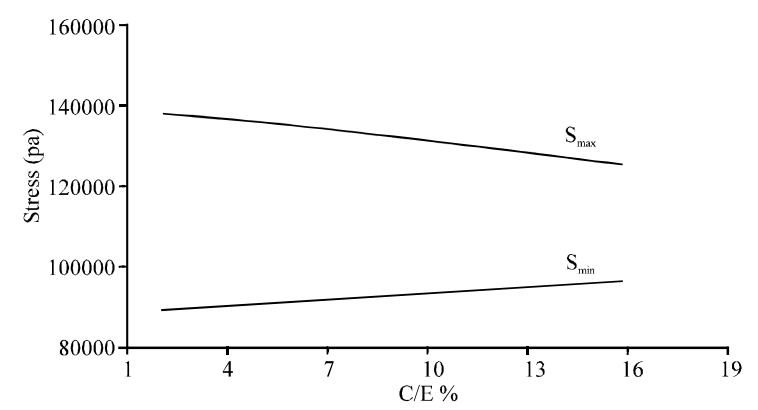

(b)

Fig. 4: Effects of viscoelastic property on: (a): stress profile across the arterial wall (b): maximum and minimum of stress pulse

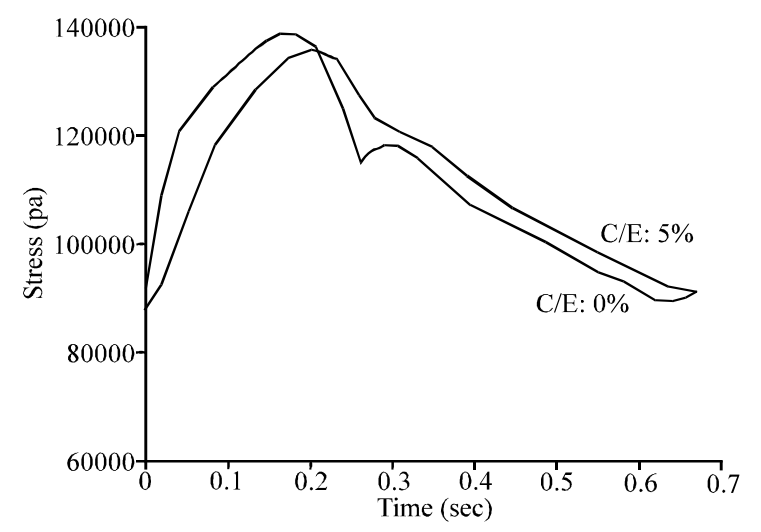

Fig. 5: Effects of viscoelastic property $(\mathrm{C} / \mathrm{E}=5 \%)$ on stress wave form

resultant stress wave. With significant increase of $\mathrm{C} / \mathrm{E}$, the presence of the second peak in the stress wave and also the sharp rise and consequent decrease in the wave almost vanish leading to a smoother stress pulse.

Effects of hypertension: Figure 6 compares stress waves resulting from two types of pressure waves: normal and hypertensive aortic pressure waves for models with $\mathrm{E}=300 \mathrm{kPa}$ and non-voscoelastic (Fig. 6a)

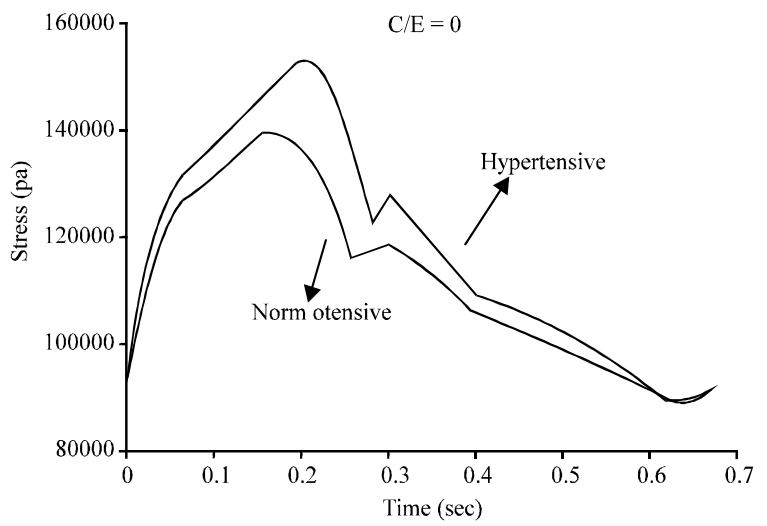

(a)

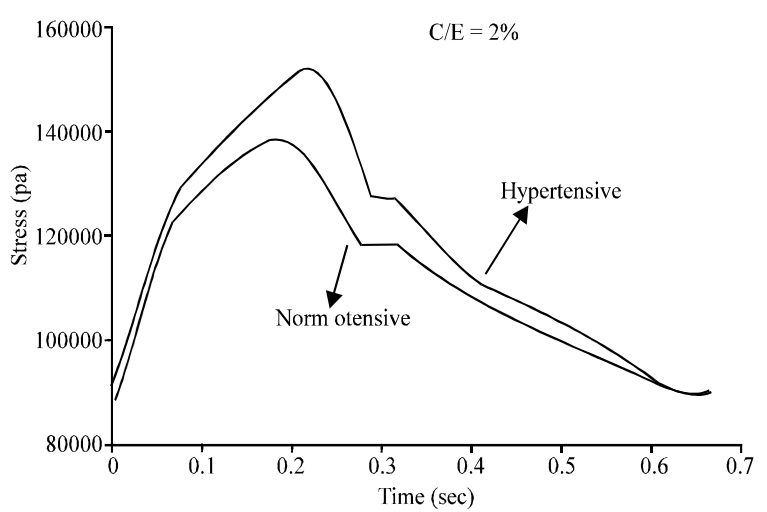

(b)

Fig. 6: Effects of hypertension on stress wave for models without (a): and with (b): viscoelastic properties

and viscoelastic (Fig. 6b) properties, respectively. For the model with hypertensive pressure waveform the $9 \%$ increase of systolic pressure compared to the model with normal pressure waveform, results in a proportional increase in maximum stress values in the absence of viscoelasticity. For a hypertensive subject not only a higher maximum stress value is evident, but also a sharper waveform exists. For models with viscoelastic parameter $(\mathrm{C} / \mathrm{E}=2 \%)$ the excess of maximum stress in a hypertensive subject compared to a normal subject reduces to $8 \%$ compared with nonviscoelastic models as indicated in Fig. 6b. This Figure shows that even for a small viscoelastic parameter this effect leads to the smoothness of stress waveform and omission of second peak.

The oscillatory nature of blood pressure causes pulsatile stress in the arterial wall. Such stresses are maximized on the Intima, resulting in tensile stretch of the endothelial cells. The excessive stress causes endothelial damage and development of atheroma in the 
arterial wall ${ }^{[9,21]}$. Results of this study show that the stress values are markedly dependent on the pressure value and waveform, together with the mechanical properties of the arterial wall.

Pulsatile stress induced by cyclic load is determined by mechanical fatigue. There are four major determinants in fatigue process: (i) maximum stress value, (ii) stress wave amplitude, (iii) the ratio of $S_{\min }$. to $S_{\max }$ (R) and (iv) stress waveform. Results show that all four parameters are influenced by the research variables.

The pressure waveform is a major parameter affecting the stress pattern in arterial wall. Hypertensive arteries result in higher and sharper stress waves not only because of a higher systolic value but also because of the sharper waveform. The hypertensive pressure pulse leads to a sharp and elevated maximum stress value of stress wave. Such wave may result in more severe consequences in the fatigue process, which could result in degeneration and fragmentation of elastin fibers of the media and endothelial damage of Intima. The effect of viscoelasticity is to reduce the peak stress and smoothening of the stress waveform. This parameter also decreases the amplitude of the stress pulse and increase of the ratio of $S_{\min } / S_{\max }$ by increase of minimum stress value.

Higher elastic modulus results in higher stress values. Since aging results in stiffer arteries it also leads to higher stress values. The combination of these parameters produces the arterial wall stress pattern. For example, combination of aging and hypertension would lead to acceleration of process of mechanical arterial degeneration and plaque formation. Arterial wall opposes the trend of sever consequences by adaptation and remodeling ${ }^{[21]}$. Such phenomena include geometrical and mechanical alterations for reduction of pressure induced pulsatile stress.

\section{CONCLUSION}

Dynamic stress analysis of the arterial wall can provide improved characterization of arterial wall mechanics as a determinant of the arterial wall pathology. Of the different mechanical parameters effecting arterial wall stress distribution, wall elastic modulus and viscoelastic parameter are found to be significant. Pressure waveform is also a significant parameter in determining pulsatile stress. The pulsatile stress of the arterial wall is an appropriate descriptor of effects of aging and hypertension, through the elevated pressure wave and altered mechanical parameters.

\section{REFERENCES}

1. Nichols, W.W. and M.F. O'Rourke, 1998. McDonald's blood flow in arteries, theoretical, experimental and clinical principles, 4th Edn., Arnold.

2. Humphrey, J.D., 2002. Cardiovascular solid mechanics, Springer-Verlag.

3. Nerem, R.M. et al., 1994. The regulation of vascular endothelial biology by flow. In: Cell Mechanics and Cellular Engineering, SpringerVerlag.

4. Sho, E. et al., 2003. High flow drives endothelial cell proliferation during flow-induced arterial remodeling associated with the expression of vascular endothelial growth factor. Exp. Mol. Pathol., 75: 1-11.

5. Honda, H.M. et al., 2001. A complex flow pattern of low shear stress and flow reversal promotes monocyte binding to endothelial cells. Arteriosclerosis, 158: 385-390.

6. Buchanan, J.R. et al., 2003. Hemodynamics simulation and identification of susceptible sites of atherosclerotic lesion formation in a model abdominal aorta. J. Biomech., 36: 1185-1196.

7. Thubrikar, M.J. and F. Robicsek, 1995. Pressureinduced arterial wall stress and atherosclerosis. Ann. Thorac. Surg., 59: 1594-1603.

8. Vito, R.P. and S.A. Dixon, 2003. Blood vessel constitutive models-1995-2002. Ann. Rev. Biomed. Eng., 5: 413-439.

9. Gao, F., M. Watanabe and T. Matsuzawa, 2006. Stress analysis in a layered aortic arch model under pulsatile blood flow. Biomed. Eng. Online, 5: 25.

10. Thubrikar, M.J. et al., 1990. Study of stress concentration in the walls of the bovine coronary arterial branch. J. Biomech., 23 (1): 15-26.

11. Fung, Y.C. and C.J. Chuong, 1983. Threedimensional stress distributions in arteries, ASME, 105: 268-274.

12. Loree, H.M. et al., 1992. Effects of fibrous cap thickness on peak circumferential stress in model atherosclerotic vessels. Circ. Res., 71 (4): 850-57.

13. Lehoux, S. and A. Tedgui, 2000. Cellular mechanics and gene expression in blood vessels. J. Biomech., 36: 631-643.

14. Brown, T.D., 2000. Techniques for mechanical stimulation of cells in vitro: A Rev. J. Biomech., 33: 38 .

15. Stamenovic, D. and N. Wang, 2000. Cellular responses to mechanical stress invited review: Engineering Approaches to Cytoskeletal Mechanics. J. Appl. Physiol., 89: 2085-2090. 
16. Bruce, M.R. et al., 1998. Skeletal tissue mechanics. New York: Springer-Verlag.

17. Armentano, et al., 1995. Arterial wall mechanics in conscious dogs. Circulation Res., 76: 468-478.

18. Nakashima, T. and J. Tanikawa, 1971. A study of human aortic distensibility with relation to atherosclerosis and aging. Angil., 22 (8): 477-490.

19. O'Rourke, M.F., R.P. Kelly and A.P. Avolio, 1992. The arterial pulse, Lea and Febiger.
20. Murgo, J.P. et al., 1980. Arterial input impedance in normal man: Relationship to Pressure Wave Forms, Circulation, 62 (1): 105-116.

21. Niroomand, H., M. Tafazoli and F. Ghalichi, 2007. Flow characteristics in elastic arteries using a fluidstructure intraction model. Am. J. Applied Sci., 4 (8): 516-524. 